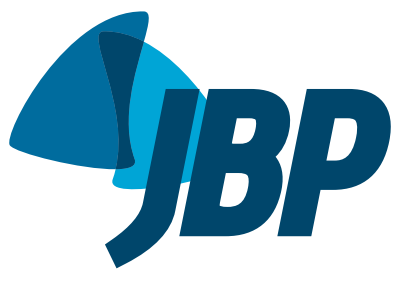

\title{
Probe-based confocal laser endomicroscopy of the airways: physiological and pathological characteristics of preneoplastic and neoplastic lesions
}

\author{
Juliana Rocha Mol Trindade, ${ }^{1, a}$, Viviane Rossi Figueiredo ${ }^{2, b}$, \\ Paulo Manuel Pêgo-Fernandes ${ }^{3, c}$, Ricardo Mingarini Terra ${ }^{1, d}$
}

\section{TO THE EDITOR:}

Primary lung cancer has a high incidence and high mortality, with a five-year survival rate of $10-15 \%$. That is because most patients are diagnosed at advanced stages of the disease. Various imaging methods and histological sampling methods have been developed to enable earlier and more precise diagnosis of lung cancer. ${ }^{(1)}$

Although it is feasible to obtain airway tissue samples via bronchoscopy, there is great interest in researching methods that will allow in vivo microscopic visualization of tissue structure. That is because of the constant need to differentiate malignant or premalignant lesions from the normal mucosa, making it possible, if not to establish a diagnosis, then to guide biopsy to ensure that the sample collected is representative of the lesion.

The advent of lasers led to the development of an imaging technique known as probe-based confocal laser endomicroscopy (pCLE), which uses a miniprobe (containing thousands of optical fibers and connected to a laser light source) to analyze small areas of tissue, allowing microscopic changes (in the mucosa and other tissues) to be assessed. (2) Some studies have defined the characteristics of endomicroscopy images of airway structures (such as the trachea, bronchi, and alveoli), as well as those of certain diseases (such as some types of lung cancer). (3)

One pCLE system currently on the market is the Cellvizio system (Mauna Kea Technologies, Paris, France), which uses a wavelength of $488 \mathrm{~nm}$ : images result from autofluorescence of subepithelial elastin fibers in the bronchial mucosa, which account for more than $50 \%$ of airway support tissue.

A prospective study of four cases was conducted at the University of São Paulo School of Medicine Hospital das Clínicas. The study was approved by the local research ethics committee (Protocol no. 315/13).

The patients evaluated were suspected of having centrally located lung cancer, information that is important for access to the tumor under direct visualization and correct positioning of the miniprobe, which should be in contact with the lesion. Of the four patients, two were male. Three of the patients were smokers (mean smoking history, 58 pack-years). The following histological types were identified: adenocarcinoma, in two; and squamous cell carcinoma, in two. Initially, we used endoscopy (flexible bronchoscopy) to assess the entire airway, identifying any visible changes. We then inserted the PCLE miniprobe into the working channel of the bronchoscope, positioning the miniprobe sequentially over normal mucosa of the tracheobronchial tree and lung parenchyma, subsequently placing it on the surface of the tumor. Figure 1 shows an example of endoscopic visualization of the pCLE miniprobe and identification of a lesion in the orifice of the right upper lobe.

The literature suggests that analysis of PCLE images allows identification of five patterns of distribution of elastin fibers in the airway submucosa. ${ }^{(4)}$ These patterns vary according to their location in the airway, and, even in our initial experience, it was possible to identify these five patterns in all cases studied: in the trachea, carina, and main bronchi, elastin fibers are parallel to each other, forming a single longitudinal layer (Figure $2 \mathrm{~A}$ ); in the secondary carina, elastin fibers are parallel to each other in layers, which are distinguished by the orientation of the fibers. At that time, it was also possible to perceive the orifice between the bronchial gland and the airway (Figure 2B); in the segmental and subsegmental bronchi, there is greater rarefaction of the elastin fibers, which begin to interlace with the underlying layers (Figure 2C); in the distal bronchioles, the network of interlaced elastin fibers of the bronchi assumes a helical conformation, culminating in the formation of a network of support for the alveolar bronchioles (Figure 2D); and the alveolar bronchioles and alveoli are represented by an elastin fiber network that expands and forms a three-dimensional support framework, comprising structures such as sacculations for the support of the alveolar cells and capillaries (Figure 2E).

In addition to the normal patterns, we visualized changes in the alveoli of the patients who smoked, marked by alveolar filling with inflammatory cells (Figures $2 \mathrm{~F}$ and $2 \mathrm{G})$, well as changes in the pulmonary parenchymal architecture in peripheral areas of the tumor, characterized by alveolar wall thickening secondary to an increase in the elastin bundle diameter, alveolar edema, and a 
large number of macrophages in the alveolar space (Figures $2 \mathrm{H}$ and $2 \mathrm{~J}$ ).

In the cases of adenocarcinoma, it was possible to see, in the tumor area, an amorphous mass filling the alveolar space and destroying its wall, characterized by alternating areas of high fluorescence, due to the misshapen aggregate of elastin, and dark cavities, representinglarge blocks of tumor cells, which are not autofluorescent (Figures $2 \mathrm{~K}$ and $2 \mathrm{M}$ ). In contrast, in the cases of squamous cell carcinoma, we saw an amorphous mass with branches that were less fluorescent, thicker, and more numerous, representing stroma, as well as dark layers representing the cellular component (Figures $2 \mathrm{~N}$ and 20).

On the basis of knowledge of the usual distribution of elastin fibers in each airway segment, studies

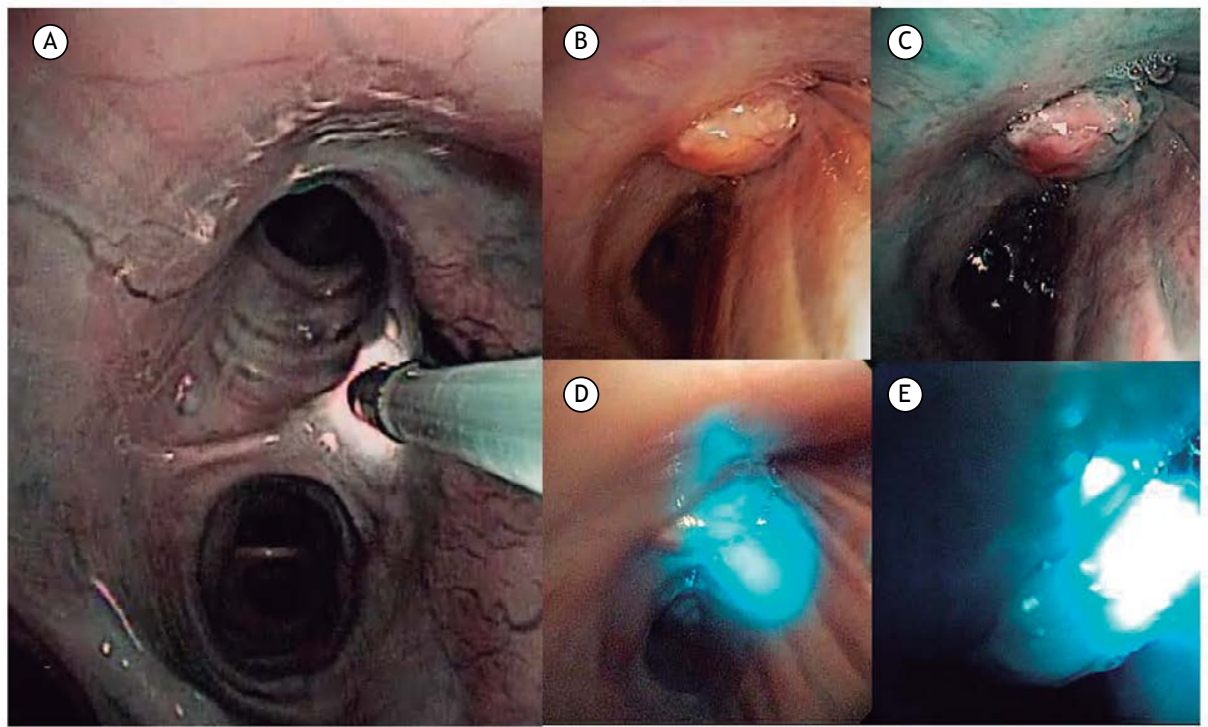

Figure 1. In A, endoscopic visualization of the confocal endomicroscopy miniprobe; in $B$; white-light endoscopic image of a neoplastic lesion; in C, narrow-band image of a neoplastic lesion; in B, illumination by the laser light source in the airway; and in $\mathrm{E}$, contact of the miniprobe with the laser light source for generation of confocal endomicroscopy images.
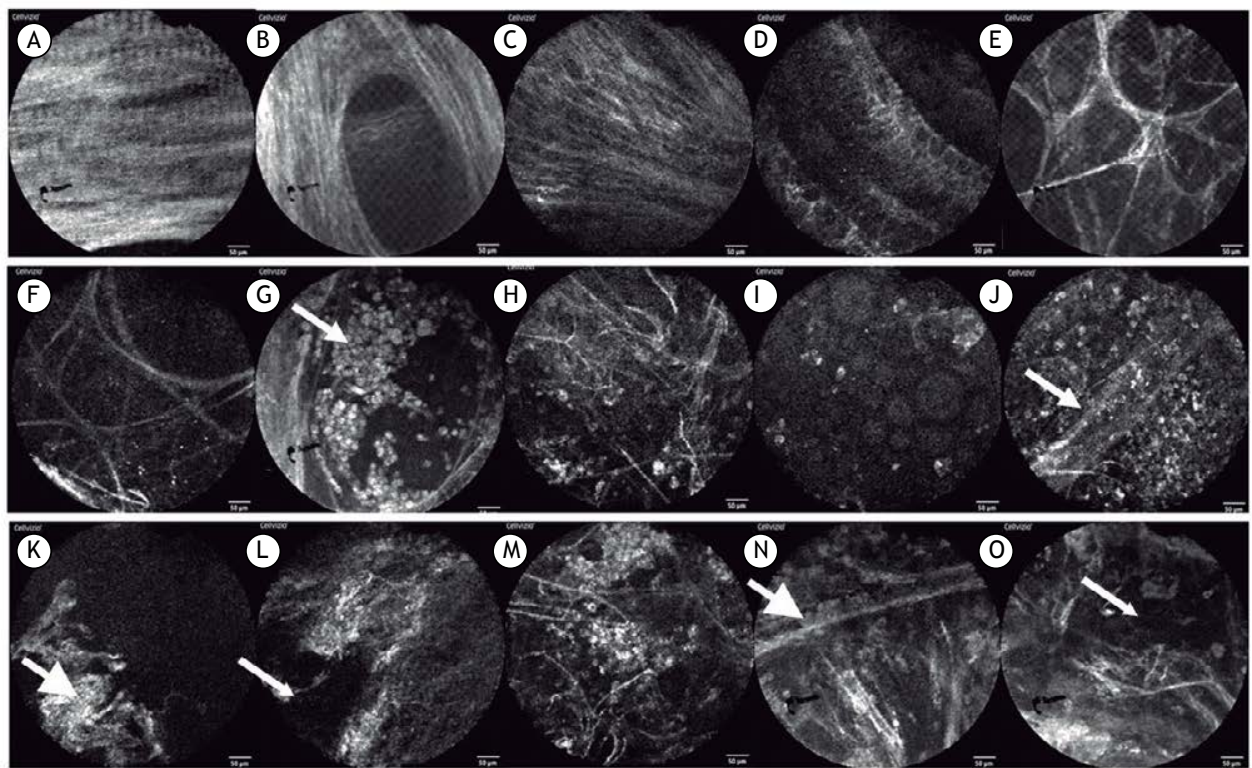

Figure 2. Distribution of elastin fibers in the airways. In $A$, trachea; in $B$, bronchial gland; in $C$, segmental bronchus;

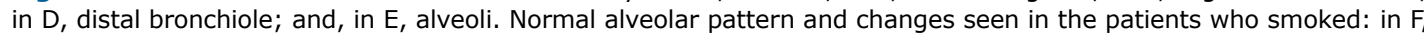
normal alveolar architecture; and, in G, pulmonary alveoli filled with inflammatory cells and alveolar macrophages (arrow). Inflammatory changes in the area surrounding the tumor: in $\mathrm{H}$, alveolar wall destruction associated with disorganized proliferation of elastin fibers, as well as elastin fiber thickening; in I, alveolar edema, caused by filling of the alveolar space with fluid; and, in J, a large quantity of cells in the alveoli, making it possible to visualize an alveolar capillary (arrow). Changes in the tumor: in K, L, and M, a hyperfluorescent aggregate of elastin (large arrow) alternating with a dark area corresponding to a neoplastic cell conglomerate (small arrow); and, in $\mathrm{N}$ and $\mathrm{O}$, an amorphous, less autofluorescent mass (stroma: large arrow), alternating with a dark area of hypercellularity, and tumor cell conglomeration (small arrow). 
have been carried out to standardize the changes in these structures, comparing them with those in samples obtained from surgical specimens, and thus correlate them for the in vivo diagnosis of malignant and premalignant lesions. ${ }^{(6)}$

It is believed that pCLE is useful in the diagnosis and follow-up of numerous airway diseases other than central neoplasms. Many research centers have been conducting tests to assess the applicability of pCLE in non-neoplastic diseases, which includes the diagnosis and reevaluation of patients with alveolar proteinosis, detecting filling of the alveolar space and the need for lung lavage(7); the follow-up of lung transplant recipients, it being possible to detect early graft rejection ${ }^{(8)}$; the diagnosis of pneumonia, such as that induced by administration of amiodarone ${ }^{(9)}$; infection with Pneumocystis jirovecii in HIV-infected patients ${ }^{(10)}$; and even the diagnosis of pulmonary fibrosis.

Although pCLE is still in development, it is a method that is quite promising. Microscopic assessment of the airways and lung parenchyma without the need for tissue collection for histopathological examination is among the main advantages of pCLE, given that it reduces the risk inherent in any biopsy method, minimizing the risk of complications for patients requiring a diagnosis of lung diseases. However, further studies are needed in order to confirm the applicability and reproducibility of pCLE for clinical use instead of the methods currently in use, as well as to quantify the cost reduction resulting from its use.

\section{REFERENCES}

1. Streba $C T$, Gîltan $A M$, Gheonea $I A$, Demetrian $A$, Soimu AV, Săftoiu $A$, et al. Utility of confocal laser endomicroscopy in pulmonology and lung cancer. Rom J Morphol Embryol. 2016;57(4):1221-1227.

2. Thiberville L, Salaun M, Lachkar S, Dominique S, Moreno-Swirc S, Vever-Bizet $C$, et al. Confocal fluorescence endomicroscopy of the human airways. Proc Am Thorac Soc. 2009;6(5):444-9. https://doi. org/10.1513/pats.200902-009AW

3. Yserbyt J, Dooms C, Ninane V, Decramer M, Verleden G. Perspectives using probe-based confocal laser endomicroscopy of the respiratory tract. Swiss Med Wkly. 2013;143:w13764. https://doi. org/10.4414/smw.2013.13764

4. Thiberville L, Moreno-Swirc S, Vercauteren T, Peltier E, Cavé C, Heckly GB. In vivo imaging of the bronchial wall microstructure using fibered confocal fluorescence microscopy. Am J Respir Crit Care Med. 2007;175(1):22-31. https://doi.org/10.1164/rccm.2006056840C

5. Wellikoff AS, Holladay RC, Downie GH, Chaudoir CS, Brandi L, Turbat-Herrera EA. Comparison of in vivo probe-based confocal laser endomicroscopy with histopathology in lung cancer: A move toward optical biopsy. Respirology. 2015;20(6):967-74. https://doi. org/10.1111/resp. 12578

6. Sorokina A, Danilevskaya O, Averyanov A, Zabozlaev F, Sazonov $D$, Yarmus $L$, et al. Comparative study of ex vivo probe-based confocal laser endomicroscopy and light microscopy in lung cancer diagnostics. Respirology. 2014;19(6):907-13. https://doi.org/10.1111/ resp. 12326

7. Danilevskaya O, Averyanov A, Lesnyak V, Chernyaev A, Sorokina A. Confocal laser endomicroscopy for diagnosis and monitoring of pulmonary alveolar proteinosis J Bronchology Interv Pulmonol. 2015;22(1):33-40. https://doi.org/10.1097/LBR.0000000000000126

8. Yserbyt J, Dooms C, Decramer M, Verleden GM. Acute lung allograft rejection: diagnostic role of probe-based confocal laser endomicroscopy of the respiratory tract. J Heart Lung Transplant. 2014;33(5):492-8. https://doi.org/10.1016/j.healun.2014.01.857

9. Salaün M, Roussel F, Bourg-Heckly G, Vever-Bizet C, Dominique S, Genevois A, et al. In vivo probe-based confocal laser endomicroscopy in amiodarone-related pneumonia. Eur Respir J. 2013;42(6):1646-58. https://doi.org/10.1183/09031936.00191911

10. Shafiek H, Fiorentino F, Cosio BG, Kersul A, Thiberville L, Gómez $C$, et al. Usefulness of Bronchoscopic Probe-Based Confocal Laser Endomicroscopy in the Diagnosis of Pneumocystis jirovecii Pneumonia. Respiration. 2016;92(1):40-7. https://doi. org/10.1159/000447431 